\section{Trabajo de parto}

El inicio del trabajo de parto representa un momento muy particular en la secuencia de la reproducción humana. Un parto dura, en promedio, solo $2 \%$ del tiempo total del embarazo. En un lapso tan breve, el feto, cuyo crecimiento y desarrollo lo habilitan para vivir fuera del antro materno, debe afrontar los fenómenos agudos que facilitan su salida a través del canal del parto. El control de la normalidad de su evolución es la tarea del equipo de salud.

El parto normal está caracterizado por una iniciación espontánea, una evolución eutócica y una terminación natural. Desde el punto de vista de la evolución, el trabajo de parto puede dividirse en tres etapas. La primera se conoce como fase latente, la segunda como fase activa æ (ambas constituyen el período de dilatación) y la última, fase expulsiva o período expulsivo (el alumbramiento constituye una cuarta etapa). Aunque no hay certeza acerca de la duración de cada fase, se acepta que los extremos superiores de duración de cada fase son los siguientes: la latente hasta 8 horas (de 0 a $3 \mathrm{~cm}$ de dilatación cervical), la activa hasta 10 horas (de 3 a $10 \mathrm{~cm}$ de dilatación cervical) y el período expulsivo hasta 2 horas (desde la dilatación completa hasta la expulsión fetal). Sin embargo, hay que tomar en cuenta que existen diferencias importantes en estos valores ya sea que se trate de parturientas nulíparas o multíparas?

\section{Reconocimiento del inicio del trabajo de parto}

En general, se acepta que el parto comienza cuando existe una actividad uterina rítmica, con una frecuencia mínima de 2 contracciones cada 10 minutos, e intensidad de ++, un cuello uterino borrado $80 \%$ y con $2 \mathrm{~cm}$ de dilatación. Sin embargo, lo que parece tan fácil, no lo es.

La embarazada es la que primero sospecha el diagnóstico por la aparición de contracciones uterinas regulares y dolorosas, con mayor o menor brusquedad. Con frecuencia, éstas se encuentran precedidas por pérdida de limos (moco cervical) con algunas estrías de sangre o intensamente teñido por ella, y otras veces por la rotura de las membranas ovulares; con menor frecuencia, puede aparecer diarrea debida a la estimulación intestinal por prostaglandinas o polaquiuria por el grado de encaje del polo cefálico.

Durante la fase latente, el diagnóstico de trabajo de parto es uno de los más difíciles en obstetricia y el médico o la obstétrica son los que deben confirmarle a la embarazada que esta primera etapa del final del embarazo comenzó. Muchas veces se requieren dos evaluaciones realizadas por un mismo médico y separadas por un intervalo de dos horas, para establecer el progreso de la dilatación y el borramiento del cuello.

El problema no sería tan importante si no estuviera vinculado con la decisión de ingresar o no ingresar la embarazada al hospital. Un ingreso muy anticipado (en fase latente), aumenta el displacer materno y el número de intervenciones no justificadas ${ }^{3}$. Una solución está en los programas docentes diseñados para ayudar a las embarazadas a reconocer los signos correspondientes a un franco trabajo de parto. Esta intervención está asociada con una reducción importante en el número de consultas que hacen estas embarazadas por un falso trabajo de parto ${ }^{4}$.

La relación entre el estado del cuello uterino y la iniciación del parto es muy conocida. El cervix se caracteriza por la evaluación de cuatro parámetros: borramiento, dilatación, consistencia, posición y de un quinto a considerar: altura de la presentación. Bishop diseñó una puntuación dándole de 0 a 3 puntos a cada una de las variables mencionadas (Cuadro 1); a medida que la puntuación aumenta, menor es el tiempo que resta al nacimiento. No todas las variables elegidas tienen la misma relación con ese intervalo, sin embargo, la puntuación de Bishop es todavía el mejor y el más simple método para determinar la evolución del trabajo de parto ${ }^{5}$.
Cuadro 1: puntuación de Bishop.

\begin{tabular}{lllll}
\hline Condición cervical & \multicolumn{5}{l}{ Puntuación } \\
\hline & 0 & $\mathbf{1}$ & $\mathbf{2}$ & $\mathbf{3}$ \\
\hline Borramiento $(\%)$ & $0-30$ & $40-50$ & $60-70$ & 80 \\
\hline Dilatación (cm) 0 & $1-2$ & $3-4$ & $5-6$ & \\
\hline Altura de la presentación & -3 & -2 & -1 ó 0 & +1 ó +2 \\
\hline Consistencia & firme & medio & blando & - \\
\hline Posición & posterior intermedia & anterior & - \\
\hline
\end{tabular}

De Bishop EH. A pelvic scoring for elective induction. Obstet Gynecol, 1974; 24: 266

Además de ser difícil de establecer un momento ideal para ingresar una paciente en el ámbito hospitalario, éste será diferente para cada mujer. El más apropiado pareciera ser aquél en el que la embarazada se encuentra más reconfortada dentro del hospital que fuera de él y, por lo tanto, una buena costumbre sería requerir su opinión en esta circunstancia y ponerla en el contexto de su condición económica, educativa y familiar para equivocarnos lo menos posible.

\section{Control del trabajo de parto}

Cuando la embarazada ingresa, es importante analizar su estado físico y psicológico, ambos tan importantes como el progreso mismo de la dilatación cervical o el descenso de la presentación.

\section{1.- Apoyo a la parturienta:}

Existe evidencia de que la presencia de un acompañante especialmente adiestrado (doula) para estar con la parturienta durante todo el trabajo de parto y el parto, disminuye la necesidad de analgesia, episiotomía, operaciones cesáreas, incrementa la sensación placentera de las madres y aumenta la frecuencia de lactancia natural ${ }^{6-7}$. Gagnon y col ${ }^{8}$ mostraron el efecto beneficioso de las enfermeras ocupando el papel de las doulas en reducir la necesidad de la estimulación de la contractilidad uterina en 17\% (RR 0,83 IC95\% 0,67-1,04). En Argentina no se dispone de este tipo de mujeres preparadas para dar apoyo emocional y físico, ofrecer información de manera continua y favorecer el inmediato contacto entre la madre y su hijo recién nacido. Tampoco posee suficiente cantidad de enfermeras para cubrir individualmente el total de los partos. En general se permite pasar a la llamada sala de "dilatante" a un familiar de la parturienta (casi siempre el padre del niño por nacer) aunque su preparación para enfrentar la circunstancia rara vez es la adecuada. La presencia de otros hijos en ese lugar todavía se discute y la respuesta al problema es muy variada, pero, no existe ninguna evidencia científica que avale cualquiera de ellas. Quizá fuera bueno preguntarle a la madre, en la consulta prenatal, quién o quienes ella quiere que la acompañen en ese momento.

Cuando el personal a cargo del cuidado de la parturienta se dirige a ella, suele hacerto tlamándola: "mamita"-, "mami", "madrecita", "querida"; todas reciben el mismo nombre, les guste o no les guste. Una buena costumbre es que, al ingresar a la maternidad, la embarazada tuviera la oportunidad de manifestar como el personal debe dirigirse a ella y también conviene ofrecerle ambientes con la mayor privacidad posible, contestar todas sus dudas con información verdadera y solicitarles su opinión cuando haya más de una opción para su cuidado.

La falta de estudios aleatorizados que demuestren las ventajas del parto hospitalario sobre el domiciliario para embarazadas de bajo riesgo, hace que sea imprescindible cuidar que el cambio al ingresar al ambiente hospitalario no se convierta en un daño para la madre, en cambio del beneficio que pretendemos los médicos. 


\section{Medidas generales:}

En las distintas instituciones donde se asisten partos suelen existir las denominadas rutinas para el control periódico de la presión arterial, el pulso y la temperatura corporal, sin embargo, no existen evidencias sobre los beneficios que ellas aportan. En lugar de establecer horarios rígidos para los controles, estos debieran establecerse según la necesidad de cada embarazada ${ }^{9}$.

A pesar de que en muchas instituciones todavía se utiliza administrar un enema y rasurar la región vulvoperineal, la evidencia proveniente de ensayos clínicos controlados ponen de manifiesto su falta de efectividad. El enema se administra con el objeto de facilitar el descenso de la presentación, estimular las contracciones uterinas y disminuir la contaminación al momento del nacimiento. Los estudios randomizados muestran que el único efecto es el de disminuir la aparición de materia fecal al momento del período expulsivo, algo que más que afectar la salud, afecta la estética. La mayor parte de las madres que no reciben la intervención expresan su satisfacción por ese hecho. Esta costumbre debe erradicarse de las maternidades, salvo que sea la propia parturienta la que la solicite ${ }^{10}$.

Dos antiguos trabajos (1922 y 1965) $11-12$, presentaron evidencia que cuestionaba las supuestas ventajas del rasurado vulvar 11, disminuir el riesgo de infección vulvoperineal y hacer que la sutura de las heridas de la región sean más fáciles y seguras. La mayoría de las madres se muestran agradecidas si no se las rasura, por lo que el procedimiento no debería, salvo que sea la parturienta la que lo solicite.

También es motivo de discusión la alimentación e hidratación durante el trabajo de parto ${ }^{12}$. La restricción absoluta intenta evitar el reflujo gástrico si la embarazada debe someterse a una intervención con anestesia general. Administrar medicación para restringir el volumen gástrico o incrementar el $\mathrm{pH}$ del contenido, no son efectivas. La conducta más razonable es la de hidratar y alimentar a la madre moderadamente y evitar el uso de anestesia general ya que si bien la aspiración de contenido gástrico es un accidente raro, la enfermedad que produce es grave.

\section{Control de la contractilidad uterina:}

Desde el punto de vista clínico, cuatro son las características de la contractilidad uterina que deben controlarse: tono, intensidad, duración e intervalo. La palpación abdominal es suficiente para controlar las características mencionadas en el parto de bajo riesgo y tiene la gran ventaja de permitir la deambulación de la parturienta y la adopción de la posición más cómoda.

De las cuatro características, dos se miden de manera cualitativa (tono e intensidad) y dos cuantitativa (duración e intervalo). El tono se evalúa entre contracciones uterinas y se considera normal cuando el útero se deprime con facilidad a la compresión digital y las partes fetales se reconocen con facilidad a la palpación. Cuando el tono se eleva empiezan a perderse estas cualidades. La intensidad de las contracciones uterinas se aprecia en su pico y se valora en cuatro cruces, según la posibilidad de deprimir el útero (+) o la sensación de consistencia pétrea (++++). La duración de la contracción (tiempo entre la captación de su inicio hasta la desaparición de esa sensación) varía entre 15 y $60 \mathrm{seg}$. Habitualmente, el intervalo entre contracciones se mide entre el final de una contracción y el comienzo de la otra, sin embargo, esta medida resulta más complicada que la de su frecuencia (número de contracciones en $10 \mathrm{~min}$ ) cuya exactitud, además, es mayor. Esta característica varía entre 2 contracciones cada $10 \mathrm{~min}$ (al comienzo del trabajo de parto) y 5 contracciones (final del período de dilatación y el expulsivo) cada $10 \mathrm{~min}$.

La frecuencia de los controles se establece, de manera ar- bitraria, en uno cada hora al comienzo del período de dilatación, uno cada media hora a partir de los $5-6 \mathrm{~cm}$ de dilatación cervical, hasta hacerse continuo durante el período expulsivo. No hay estudios que muestre la utilidad de este esquema, así como el de cualquier otro.

\section{4.- Control de la frecuencia cardíaca fetal (FCF):}

Este es el control de salud fetal más importante ya que de las variaciones que se detectan dependen muchas indicaciones de terminación artificial del trabajo de parto. El grupo de investigadores liderado por R. Caldeyro Barcia13-14, describió, entre 1958 y 1964, las variaciones de la FCF vinculadas con la compresión de la cabeza fetal, el cordón umbilical y la hipoxia fetal15. En ese mismo período, los resultados obtenidos por investigadores europeos y estadounidenses apoyaban las conclusiones de la escuela uruguaya 16 .

\section{a) Auscultación intermitente}

Se realiza con el estetoscopio de Pinard o con alguna de sus modificaciones y resulta ser una forma de control suficiente para el control de los partos de bajo riesgo. El criterio para diagnosticar sufrimiento fetal con este método se basa en los límites de la normalidad de la FCF: un máximo de 160 lat/min y un mínimo de 100-120lat/min, por fuera de estos límites se admite que el feto sufre de hipoxia.

También se han popularizado los aparatos portátiles que permiten la auscultación de los latidos fetales por ultrasonido; estos tienen la ventaja de poder tomar la FCF durante la contracción uterina, lo que no es posible con el de Pinard. Con ellos, una auscultación cuidadosa y realizada por personal entrenado podrá identificar los dips II o tardíos y los de compresión de cordón, ambos relacionados con el diagnóstico de sufrimiento fetal agudo. Si es posible, cuando se está frente a un diagnóstico de sufrimiento fetal con esta forma de auscultación, se debe recurrir al monitoreo contínuo de la FCF.

\section{b) Auscultación continua}

Los aparatos utilizan los ultrasonidos o la señal de la onda R del ECG fetal para calcular la llamada frecuencia cardíaca instantánea y registrarla de manera continua en un papel. Tiene varias ventajas: identificar pequeñas variaciones de la FCF, medir los tiempos entre el valle de un dip y el pico de la contracción uterina, medir la duración y la magnitud de la caída y conservar el registro de lo sucedido con la FCF en distintos momentos del trabajo de parto. Cuando se utiliza este método se corre el riesgo de tomar conductas agresivas no justificadas debido al elevado número de resultados falsos positivos (baja especificidad del método $)^{17}$. Para mejorar los resultados se sugiere complementar el registro continuo de la FCF con la valoración del estado acidobásico de la sangre del cuero cabelludo fetal.

No existen evidencias de que de las dos formas de monitorear los latidos fetales, una sea mejor que la otra en términos de morbimortalidad perinatal ${ }^{18}$. Más que uno u otro método de control de la FCF, la atención que se le dedica al control de la FCF en sí, es lo que hace la diferencia.

\section{5.- Evaluación de las características del líquido amniótico}

Tanto la cantidad como la coloración del líquido amniótico son características a tener en cuenta. Observar el líquido amniótico implica romper la bolsa æsi ésta no está rotaæ o utilizar un amnioscopio, un instrumento con la forma de cono truncado que se introduce por la vagina a través del cuello uterino dilatado y por transiluminación permite la observación del líquido amniótico atrapado entre las membranas ovulares y el polo cefálico. En Argentina es un procedimiento muy poco usado. 


\section{a) Coloración}

Desde hace más de 150 años se asocia la presencia de líquido amniótico teñido de meconio con una mayor morbimortalidad perinatal, además de un incremento en el riesgo de aparición del síndrome de aspiración de líquido amniótico meconial. La presencia de meconio espeso en el líquido amniótico al comienzo del trabajo de parto representa la condición con mayor riesgo de muerte perinatal19. La emisión de meconio después del comienzo del trabajo de parto es un suceso más raro y significa un riesgo menor que la circunstancia anterior. De todas maneras, su presencia implica un más estricto control de la salud fetal durante el trabajo de parto.

\section{b) Cantidad}

Una escasa cantidad de líquido amniótico facilita la compresión del cordón umbilical y la presencia de dips de compresión de cordón (deceleraciones variables). También, cuando se rompen las membranas ovulares y no se observa salida de líquido amniótico, los estudios de observación lo asocian con presencia meconio espeso por encima del polo cefálico y, por lo tanto, sería una situación doblemente peligrosa, meconio espeso y oligohidramnios.

Tanto la presencia de meconio espeso como de oligohidramnios durante el trabajo de parto, deben considerarse situaciones de alto riesgo.

\section{6.- Control del progreso del trabajo de parto}

La contractilidad uterina va cambiando a medida que el parto progresa. Los métodos más sofisticados de medición de la contractilidad uterina (registro continuo de la presión intrauterina) deben quedar restringidos a la investigación ya que no existe evidencia de que su uso cambie los resultados del trabajo de parto.

La dilatación cervical se la considera el mejor método para el control del progreso del trabajo de parto. Aunque su estimación presenta una sustancial variabilidad, el grado de acuerdo puede mejorarse si se permite una variación de $\pm 1 \mathrm{~cm}$. Estas consideraciones deben tenerse en cuenta a la hora de valorar un enlentecimiento de la progresión de la dilatación cervical. Para la embarazada, el tacto vaginal resulta más tolerable cuando se efectúa entre contracciones, sin embargo, la dilatación cervical es máxima en el pico de la contracción uterina. No hay acuerdo en cuando es el mejor momento para valorar la dilatación cervical, pero, dado el grado de exactitud que tiene, no pareciera ser demasiado importante, por lo menos en las embarazadas de bajo riesgo.

Las características del cuello que se valoran durante el tacto son el diámetro de la dilatación, el grado de borramiento, su longitud, el grado de reblandecimiento y la dirección de su eje mayor. Además de las características del cuello, se aprovecha la ocasión para evaluar características de las membranas ovulares (integridad, forma y en caso de estar rotas, el color del líquido amniótico) y el grado de descenso de la presentación.

La velocidad de la dilatación cervical es la que fija la normalidad o anormalidad de la evolución del trabajo de parto. Philpott 20 construyó un gráfico con una línea recta que comienza en $2 \mathrm{~cm}$ de dilatación a la hora 0 hasta $10 \mathrm{~cm}$ a la hora $8(1 \mathrm{~cm}$ por hora), que representa la progresión más lenta de su población de nulíparas (percentilo 90) y que denomina línea de alerta, la progresión de la dilatación cervical a la derecha de la curva define una población con mayor riesgo de parto prolongado21-22-23. En el mismo gráfico se traza una línea paralela pero desplazada cuatro horas hacia la derecha de la primera; ésta representa la línea de acción, y señala que se debe tomar una conducta (Figura 1).

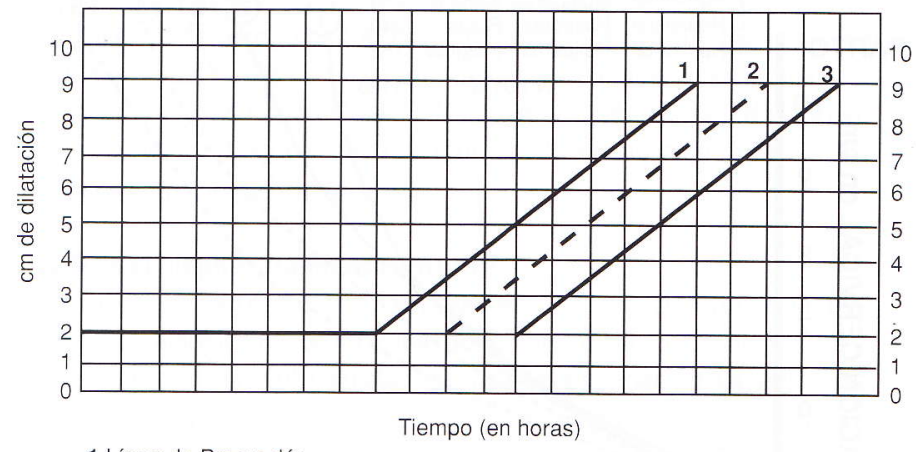

1 Línea de Progresión

2 Línea de Alerta

3 Línea de Acción

La prueba de este cervicograma mostró que $33 \%$ de curvas de dilatación cervical de las parturientas quedaban a la derecha de la línea de alerta 21 . Otro estudio realizado en México mostró que, por lo menos $20 \%$ de las curvas de dilatación de las parturientas de bajo riesgo sobrepasaban la línea de alerta durante el período de dilatación y $4 \%$ en el expulsivo. Por ello, un progreso lento de la dilatación cervical no significa anormalidad en el progreso del parto, pero debe alertar sobre la posibilidad de un problema ${ }^{24}$.

Este lento progreso puede presentarse en la fase latente 0 en la fase activa de la dilatación cervical, lo que tiene una diferencia clínica. La fase latente es poco clara; hay autores que la consideran una etapa de preparto, otros la consideran como una parte del trabajo de parto, pero, cualquiera fuera la situación, hay muy poca información acerca de su significado. Uno de los mayores problemas para los cuidados es diferenciar entre un preparto prolongado y un falso trabajo de parto. De todas maneras, cuando se considera que el preparto se prolonga, el médico a cargo del control de esa parturienta deberá tomar alguna conducta para estimular su progreso. Cuando la prolongación aparece durante la fase activa de la dilatación cervical (cruce a la derecha de la línea de acción) entonces será necesario diferenciar entre una contractilidad insuficiente 0 una desproporción cefalopélvica. En el primer caso, se recurrirá a estimular la contractilidad uterina con ocitocina, y en el segundo a efectuar una operación cesárea.

Philpott y Castle 21 trazaron una línea intermedia y paralela a las líneas de alerta y acción y la llamaron línea de derivación, con el objeto de establecer un criterio para la derivación de una parturienta que está siendo atendida en un centro no apto para realizar una operación cesárea. En el caso de que se esté asistiendo a una embarazada en este tipo de centro, esta línea es útil para monitorear la necesidad de consultar con un especialista 0 solicitar una derivación a un centro de complejidad suficiente para asistir esa complicación.

El Centro Latinoamericano de Perinatología (CLAP) de Montevideo, Uruguay, ha propuesto otro cervicograma basado en curvas obtenidas de 1.188 partos de bajo riesgo, de inicio espontáneo, evolución eutócica (sin medicación) y terminación natural y recién nacidos vigorosos y sin patología a parente. Las curvas de alerta representan el percentilo 90 de la progresión de la dilatación en esas parturientas, a la derecha de la cual se consideraría un lento progreso. Tomando en cuenta ciertas características (po- 
sición materna, paridad y estado de las membranas) derivaron cinco curvas a partir de los $4 \mathrm{~cm}$ de dilatación cervical (Figura 2).

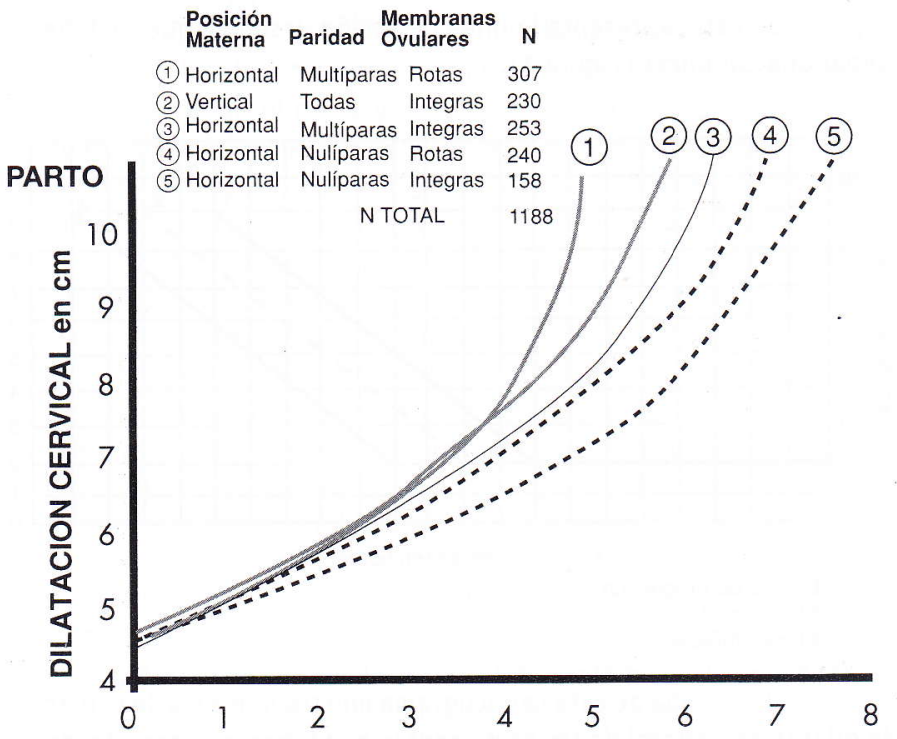

Éste es el cervicograma que sugieren las Propuestas Normativas de la Dirección de Salud Materno-Infantil del Ministerio de Salud y Acción Social de la República Argentina ${ }^{25}$. Sin embargo se desconoce que proporción de parturientas alcanzan esa línea o quedan a la derecha de ella, y cuál es la consecuencia de que ello ocurra en términos de los resultados perinatales.

El descenso de la presentación es otro parámetro para controlar durante el trabajo de parto. Este control puede realizarse por medio de la palpación abdominal, determinando cuánto de la cabeza fetal se encuentra todavía por encima del estrecho superior de la pelvis (medido en quintos de su altura que restan introducirse dentro de la pelvis); o del tacto vaginal, ya sea utilizando los planos paralelos de Hodge o los centímetros por encima y por debajo de las espinas ciáticas (de $-4 \mathrm{a}+4)$. Ninguno de los dos métodos ha sido evaluado con respecto al resultado del parto.

Es importante considerar el grado de contacto entre la presentación y el cuello uterino; la presión que ejerce la cabeza fetal sobre el cuello durante la contracción uterina está en relación con la velocidad de la dilatación cervical y esta relación es más fuerte que con la intensidad de las contracciones uterinas 27.

Es conveniente registrar todas estas evaluaciones en el partograma para hacer clara la visión del progreso del parto de cada embarazada, ya que no tiene el mismo significado el progreso de la dilatación cervical a razón de $1 \mathrm{~cm}$ por hora en una parturienta con buenas contracciones uterinas, una cabeza fetal que no desciende y con una madre que manifiesta un claro sufrimiento, que un ritmo de $0,5 \mathrm{~cm}$ por hora en otra parturienta con escasa contractilidad, con descenso de la presentación, que deambula y conversa animadamente.

\section{Dr. Omar Althabe+}

\section{Referencias}

1. Naaktgeboren C. Behavioural aspects of parturition. Animal Reprod Science, 1979; 2: 155-166.

2. Albers LL, Schiff M y Gorwada JG. The length of active labor in normal pregnancies. Obstet Gynecol, 1996; 87(3): 355-359

3. Hemminki E y Simukka R. The timing of hospital admission and the progress of labor). Eur J Obstet Gynecol Reprod Biol 1986; 22: 85-94

4. Hay-Smiyh J. Education on recognizing signs of active labour. En: Enkin MW, Keirse MJNC, Renfrew MJ, Neilson JP (eds). Pregnancy and Childbirth Module of The Cochrane Database of Systematic Reviews, 1995 (actualizada el 24/2/1995), BMJ Publishing Group, London

5. Fuentes A, Williams M. Cervical Assessment). Clin Obstet Gynecol, 1995; 38(2): 224-231

6. Hodnet ED. Support from caregivers during childbirth. En: Enkin MW, Keirse MJNC, Renfrew MJ, Neilson JP (eds). Pregnancy and Childbirth Module of The Cochrane Database of Systematic Reviews, 1995 (actualizada el 24/2/1995), BMJ Publishing Group, London

7. Langer A, Campero L, Garcia C y Reynoso S. Effects of psychosocial support during labor and childbirth on breastfeeding, medical interventions, and mother's wellbeing in a Mexican public hospital: a randomized clinical trial. Br J Obstet Gynaecol 1998; 105: 1056-1063

8. Gagnon AJ, Waghorn K y Covell C. A randomized trial of one-to-one nurse support of women in labour. Birth, 1997; 24(2): 71-77

9. Crowther C, Enkin MC, Keirse MJNC, Brown I. Monitoring the progress of labour. En: Effective care in pregnancy and childbirth. Chalmers I, Enkin MC, Keirse MJNC. (eds) 1989, Vol 2 págs 833-845. Oxford University Press, London

10. Hay-Smith J. Routine enema on admission in labour. En: Enkin MW, Keirse MJNC, Renfrew MJ, Neilson JP (eds). Pregnancy and Childbirth Module of The Cochrane Database of Systematic Reviews, 1995 (actualizada el 24/2/1995). BMJ Publishing Group, London.

11. Renfrew AJ. Routine perineal shaving on admission in labour. En: Enkin MW, Keirse MJNC, Renfrew MJ, Neilson JP (eds). Pregnancy and Childbirth Module of The Cochrane Database of Systematic Reviews, 1995 (actualizada el 24/2/1995). BMJ Publishing Group, London

12. Johnson C, Keirse MJNC, Enkin M, Chalmers I. Nutrition and hydration in Labour. En: Effective care in pregnancy and childbirth. Chalmers I, Enkin MC, Keirse MJNC. (eds) 1989, Vol 2 págs 827-832. Oxford University Press, London

13. Caldeyro Barcia R. Estudio de la anoxia fetal intrauterina mediante el EEG fetal y el registro continuo de la frecuencia cardíaca fetal, III Congreso Lat-Am Obstet Ginecol, (México), 1958. En: Cibils LA. Electronic fetal-maternal monitoring, Chapter 1: Monitoring of the mother.

14. Berdaguer PAR, Althabe 0. Monitoreo fetal. Editorial Ascune Hermanos, 1985.

15. Althabe 0, Schwarcz RL, Pose SV y col. Effects onfetal heart rate and fetal p02 of oxygen administration to the mother. Am J 0bstet Gynecol, $1967 ; 98: 858-892$.

16. Hon EH. Observations on pathologic fetal bradycardia. Am J Obstet Gynecol, 1959; 77: 1084-1091.

17. Neilson JP. EFM + scalp sampling vs intermittent auscultation in labour. En: Enkin MW, Keirse MJNC, Renfrew MJ, Neilson JP (eds). Pregnancy and Child birth Module of The Cochrane Database of Systematic Reviews, 1995 (actualizada el 24/2/1995). BMJ Publishing Group, London

18. Grant A. Monitoring the fetus, during labour. . En: Effective care in pregnancy and childbirth. Chalmers I, Enkin MC, Keirse MJNC. (eds) 1989, Vol 2 págs 846-854. Oxford University Press; London

19. Meis PJ, Hall M, Marshall JR, Hobel CJ. Meconium passage: a new calssification for risk assessment during labor. Am J 0bstet Gynecol, 1978; 131: 509-513. 20. Philipott RH. Graphic records in Labour. BMJ; 1972; 4: 163-165.

21. Philpott RH, Castle WM. Cervicographs in the management of labour in primigravidae I: the alert line to for detecting abnormal labour. J Obstet Gynaecol Br Commwlth, 1972; 79: 592-598.

22. Philipott RH, Castle WM. Cervicographs in the mamangement of labour in primigravidae. II: the action line and treatment of abnormal labour. $J 0 b s t e t$ Gynaecol Br Commwlth, 1972; 79: 599-602.

23. Dujardin B, De Schampheleire I, Sene H, Ndiaye F. Value of the alert and action lines on the partogram. Lancet, 1992; 339: 1336-1338

24. Lavender T, Alfirevic Z, Walkinshaw S. Partogram action laine study: a randomised trial. Br J Obstet Gynaecol, 1998; 105: 976-980.

25. Ministerio de Salud y Acción Social de la República Argentina. Curvas de dilatación cervical. Propuesta normativa Perinatal Tomo I, pág. 83-87, 1997.

26. Tay SK, Yong TT. Visual effect of partogram designs on the management and outcome of labour. Aust N Z Obstet Gynaecol. 1996; 36(4): 395-400

27. Allman AC, Genevier ES, Johnson MR y Steer PJ. Head-to-cervix force: an important physiological variable in labour. 2. Peak active force, peak active pressure and mode of delivery. Br J Obstet Gynaecol, 1996; 103(8): 769-775 